\title{
Germanica
}

philosophique

\section{Heidi Knörzer, Publicistes juifs entre France et Allemagne - Champions de la même cause ?}

\section{Marie Brunhes}

\section{OpenEdition}

\section{Journals}

Édition électronique

URL : http://journals.openedition.org/germanica/6231

DOI : 10.4000/germanica.6231

ISSN : 2107-0784

Éditeur

Université de Lille

Édition imprimée

Date de publication : 31 décembre 2018

Pagination : 191-193

ISBN : 978-2-913857-42-1

ISSN : 0984-2632

Référence électronique

Marie Brunhes, « Heidi Knörzer, Publicistes juifs entre France et Allemagne - Champions de la même cause? », Germanica [En ligne], 63 | 2018, mis en ligne le 31 décembre 2018, consulté le 13 janvier 2021. URL : http://journals.openedition.org/germanica/6231 ; DOI : https://doi.org/10.4000/ germanica.6231 


\section{Comptes rendus de lecture}

Heidi Knörzer, Publicistes juifs entre France et Allemagne - Champions de la même cause? Paris, Honoré Champion, 2016, 459 p.

Dans son livre Publicistes juifs entre France et Allemagne Champions de la même cause?, Heidi Knörzer met en perspective la période allant de 1848 jusqu'au déclenchement de la Première Guerre mondiale, des années au cours desquelles, du point de vue de l'histoire juive, la rupture entre monde traditionnel et partisans de la sécularisation est certes actée du fait du succès grandissant de cette dernière option tant en France qu'en Allemagne mais qui voient aussi une forme de résistance s'organiser. L'auteure propose une relecture des évènements saillants de cette période (Guerre de Crimée, Guerre de 1870-71 et lancinante question de l'Alsace-Lorraine, affaire Dreyfus...) et des préoccupations d'alors (prégnance croissante de la pensée raciale et montée de l'antisémitisme, regain ultramontain puis campagnes anticléricales en France, misère des Juifs de Russie, ambitions pour Jérusalem, formulation et essor de la proposition sioniste...) à travers la vision politique qu'en ont eue les rédacteurs des deux parutions rivales que furent l'Allgemeine Zeitung des Judentums et les Archives israélites, les deux plus importantes revues juives du XIXe siècle de part et d'autre du Rhin. Si l'objet d'étude est double et si les observations ne cherchent pas à effacer cette dualité, leur conclusion, au rebours de ce que les recherches précédentes, prises dans des logiques dichotomiques, ont souvent mis en exergue, révèle des voix à l'unisson: ces deux générations de journalistes qui ne se sont jamais rencontrés sinon au travers des débats auxquels ils se livrèrent dans «l'espace transnational» constitué par leurs revues, apparaissent sur de nombreux terrains comme des « champions de la même cause », et la fertilité de leurs échanges lamine les 
divergences qu'on aurait pu supputer ou réduit celles-ci à de simples décalages temporels dans l'appréhension des défis auxquels la minorité juive fit face de part et d'autre de la frontière.

Les noms de Ludwig Philippson (1811-1889) et d'Isidore Cahen (1826-1902) puis de Gustav Karpeles (1848-1909) et d'Hippolyte Prague (1856-1935), qui se seront tous illustrés dans d'autres fonctions (enseignement, rabbinat) et d'autres responsabilités (Philippson fait partie des premiers députés juifs des États allemands; Cahen est co-fondateur de l'Alliance israélite universelle), reviennent donc inlassablement dans cet ouvrage qui restitue en détail le fruit du travail de bénédictin auquel s'est livrée l'auteure dans les archives de ces revues, les innombrables citations empruntées à ces sources journalistiques étant systématiquement replacées dans leur contexte sociétal, politique, idéologique, en convoquant, si besoin, le discours d'autres penseurs ou activistes. En amont, Heidi Knörzer aura présenté « la génération des pères », mettant ainsi en évidence l'appartenance de Philippson et de Cahen, fils de ces maskilim, et a fortiori celle de leurs collaborateurs plus jeunes, à la modernité; de fait, ce qui unit les quatre rédacteurs est leur refus obstiné de choisir entre particularisme et universalisme, chacun d'eux revendiquant son appartenance à plusieurs identités, celle de juifs pratiquants et celle de citoyens d'un État qu'ils voudraient infailliblement moderne. Si les premiers font encore l'objet de discriminations du fait de leur judéité et continuent donc de se battre pour l'émancipation et/ou l'intégration, leurs successeurs à la tête des deux revues, des juifs ayant grandi dans le climat propice de l'abolition des dernières restrictions en France et de l'avènement progressif de l'égalité juridique en Allemagne, sont bientôt confrontés à deux périls aussi différents que la montée de l'antisémitisme et celle de l'indifférence religieuse, ce qui contribuera à faire d'eux d'ardents précurseurs du « réveil juif » (mais conduira paradoxalement au rabougrissement des Archives israélites à la taille d'une parution communautaire pourtant singulièrement ouverte dans son rapport aux frères de l'Est, évoqués fugacement par l'auteure).

L'auteure poursuit une ambition double. Il s'agit premièrement d'analyser la pensée éminemment politique de ces intellectuels engagés, ce à quoi elle s'emploie dans une maîtrise parfaite de la mécanique des transferts culturels. Ce faisant, elle prend à rebrousse-poil beaucoup de conclusions de ses prédécesseurs, nuançant et corrigeant tant les définitions schématiques et clivées auxquelles on s'était habitué - au premier chef celle de la nation (politique à la française ou ethnoculturelle à l'allemande) - que le soubassement idéel qu'on pensait définitivement stabilisé (conception de l'État; manière d'appréhender la laïcité). Le dense réseau de citations scrupuleusement renseignées fait apparaître que les discours véhiculés par ces deux revues se sont rencontrés, mutuellement influencés et fertilisés pour aboutir à un discours large- 
ment transnational, prônant en interne la « régénération » religieuse et culturelle de leurs coreligionnaires qu'il s'agit de ne pas abandonner aux radicaux rétrogrades (l'orthodoxie) pas plus qu'aux radicaux niveleurs (les réformés), régénération qui passe par une articulation de la religion avec la démocratie d'autant plus aisée pour le judaïsme qu'il est hautement moral, et préconisant en externe une vision de la citoyenneté et de la nation en « trait d'union » tentant de penser ensemble l'universel et le particulier, la citoyenneté et la différence, dans une défense des régimes politiques garantissant pleinement la liberté de conscience et se prémunissant contre toute dérive racialiste.

Le lecteur d'aujourd'hui appréciera la résonnance très actuelle que revêtent certains de leurs débats sur la réponse à offrir à l'antisémitisme, la place de la religion, la nécessité d'un service militaire, l'opportunité d'une émigration en masse des Juifs de Russie dès lors que seuls les plus riches pourraient s'offrir celle-ci. Si l'analyse des tables des matières et des éditoriaux est scrupuleuse et féconde d'enseignements, on pourra toutefois regretter que l'auteure ne se soit pas arrêtée plus longuement sur l'émergence du socialisme, que ces intellectuels représentants de la bourgeoisie libérale ne peuvent avoir manqué d'observer. Enfin, si cette étude souligne le rôle de la presse dans l'évolution vers une forme moderne de solidarité juive transnationale tout comme l'audace et l'originalité de ces journalistes face aux discours ambiants, elle renonce à explorer le retentissement de leur pensée au sein de la minorité juive et sa fertilité pour le judaïsme: si elle nous dit clairement en quoi ces publicistes ont été des juifs modernes, elle ne révèle que très peu ce qui a continué de les rattacher à la tradition.

\section{Marie Brunhes}

Ingrid Sonntag (Hrsg.), An den Grenzen des Möglichen. Reclam Leipzig 1945-1991, Berlin, Christoph Links Verlag, 2016, 544 p.

Ce livre, édité par Ingrid Sonntag, ancienne lectrice pour le Mitteldeutscher Verlag (Halle) et Kiepenheuer à Leipzig, s'attache à présenter l'histoire de la maison d'édition Reclam de 1945 à 1991. Il est composé de trois parties consacrées à l'évolution de la maison, à son programme éditorial et enfin au travail d'édition entre exigence littéraire et censure politique ; une annexe présente les contributeurs ainsi que les auteurs est-allemands mentionnés dans les contributions (malheureusement pas les lecteurs, éditeurs, membres de Reclam). C'est Wolfgang Thierse, premier citoyen de l'ex-RDA à présider le Bundestag (en 1998), qui introduit l'ouvrage en insistant sur le fait que les éditions Reclam occupaient une part importante de l'espace de communication culturel et politique en RDA. 
Les contributeurs rappellent dans la première partie le contexte historique, social et politique de l'histoire de la maison Reclam. 1946 constitue une première étape, l'obtention de la licence par l'administration militaire soviétique en mars permet une reprise de la production en prenant en compte la perte de matériel, le démontage de l'imprimerie et la dégradation qui s'en suivit au sein des bâtiments, la pénurie de papier. De nombreux dirigeants, Gotthold Müller, Hildegard Böttcher, Hans Marquardt ont tenté pendant ces quarante-six années de reprendre l'activité de la maison d'édition selon les directives de Berlin-Est tout en impulsant un nouveau départ - un aspect contradictoire souligné par Karolin Schmahl dans son article « Kontinuitäten im Neubeginn ». Directeur entre 1946 et 1953, Gotthold Müller dut aussi composer avec un autre paramètre, à savoir la coexistence avec la deuxième maison d'édition Reclam basée à Stuttgart, à laquelle une licence fut accordée en 1948. Les deux maisons d'édition n'étaient pas étroitement liées. Chacune mena au cours de ces quarante-six années sa politique éditoriale dans son contexte politique propre. La maison d'édition de Stuttgart ne fut en aucun cas une succursale de la maison d'édition de Leipzig ou vice-versa. En 1953, les deux maisons parvinrent après d'âpres discussions à un consensus : la maison-mère était à Leipzig ; les droits y restaient, toute utilisation des droits par la maison d'édition de Stuttgart devant faire l'objet d'un contrat. Ce ne fut qu'en 1991 que la fusion des maisons d'édition eut lieu.

Le programme éditorial définit le cadre dans lequel Reclam a évolué durant ces années. Reclam mit en avant trois mots d'ordre : contenu, forme, structure. Herbert Kästner montre comment Hans Marquardt, Cheflektor depuis 1953 puis Chefredakteur à partir de 1961, considérait Reclam non seulement comme une institution littéraire mais aussi comme une instance médiatrice entre littérature et beaux-arts. Pour ce qui est de la forme, il y eut d'abord un travail de refonte des collections qui entraîna une nouvelle numérotation des RUB ; de plus, on vit apparaître à la fin des années 1960 des illustrations de Horst Schuster, un des graphistes les plus reconnus en RDA ou encore en 1973 de Friedrike Pondelik, formée à l'école du graphisme. On put observer aussi une évolution au niveau du contenu. Les auteurs classiques, incontournables représentants du patrimoine culturel, furent dans les années 1940-1950 des auteurs de prédilection : Lessing, Goethe, Heine, Schiller, Mörike, Pouchkine ressortent à l'occasion de la première Foire du Livre de Leipzig rétablie en mai 1946. La poésie, peu prisée au début, prit, au fil des années, une place plus affirmée. La philosophie fit son apparition dans les années 1960 avec la publication de Ludwig Feuerbach ou Voltaire dont les écrits entraient bien dans la ligne de Berlin-Est. Les écrits de Walter Benjamin sur la littérature, au croisement entre théorie de l'art, littérature, philosophie et politique, furent publiés en 
1970 sous le titre de Lesezeichen. La maison d'édition s'intéressa aussi à la musicologie et à l'histoire de la musique, notamment avec l'édition d'une biographie de Bach qui fut traduite à travers le monde. Günter Grass fut quant à lui publié dans les années 1980. Dans son article au titre évocateur "Durchbruch durch den literarischen Schutzwall ", Konstantin Ulmer indique que Günter Grass, ignoré par Berlin-Est (et traité de «Unperson ») s'impose à partir de 1983 en tant qu'écrivain et personnage politique après avoir participé à la Berliner Begegnung zur Friedensförderung, rencontre à Berlin-Est où des artistes et des scientifiques de l'Est et de l'Ouest échangèrent sur la politique de dissuasion liée à la double décision de l'OTAN et à l'armement bilatéral.

La dernière partie de cet ouvrage brosse un panorama des auteurs publiés par les éditions Reclam pendant leurs quarante-six années d'existence. Les titres des différentes contributions sont très évocateurs et révélateurs du caractère controversé des auteurs publiés, mettant aussi en exergue la progression à petits pas dans le domaine de la publication au sein de la maison d'édition. Les années 1970 représentent une avancée dans la publication d'œuvres controversées, à l'instar de Stephan Hermlin qui dut s'imposer face à différents acteurs de la vie culturelle pour obtenir la publication de Deutsches Lesebuch. Von Luther bis Liebknecht. Les orientations de la politique culturelle énoncées lors du VIIIe congrès du Parti, "Weite und Vielfalt », semblèrent garantir une ouverture d'esprit et une sorte de libéralisation. Certains ouvrages (Briefe mit blauem Siegel ou Die wunderbaren Jahre de Reiner Kunze, Stimme Stimme de Wolfgang Hilbig) furent publiés par Reclam. Les citoyens est-allemands découvrirent aussi des classiques espagnols (Calderon, Cervantes, Luis de Gongora), français (Voltaire, Pascal, Rousseau, Zola, Balzac, Anatole France, Barbusse), des auteurs d'Amérique latine (Ernesto Che Guevara, Carlos Fuentes). Cette dernière partie nous dévoile de plus les obstacles à franchir avant publication. Fritz Mierau, par exemple, décrit dans sa contribution « Witterung für ein Scheitern » le cheminement jusqu'à la publication de la pièce de l'auteur soviétique Maïakovski Schwitzbad. La représentation de la pièce, proposant une réflexion critique sur le fonctionnement du modèle d'État et de société soviétiques, fut interdite à trois reprises $(1930,1959,1976)$, trois dates qui correspondent, selon Fritz Mierau, aux trois crises de l'appareil communiste. L'intérêt de Reclam pour la traduction de Rainer Kirsch coïncide de plus avec un mouvement d'émancipation de l'eurocommunisme marqué notamment par la prestation de Wolf Biermann à Cologne, la publication d'extraits de Alternative de Rudolf Bahro dans le Spiegel et l'incrimination de la pièce Guevara oder Der Sonnenstaat de Volker Braun à Berlin. Trois éditions de la pièce de Maïakovski seront ensuite publiées par Reclam en 1978, en 1980 et en 1982. 
Ce livre composé de cinquante-deux articles est de toute évidence une contribution particulièrement riche d'informations sur l'histoire politique et la politique culturelle de la RDA et d'éléments critiques sur les années 1946 à 1991.

Cathy Guiffroy

L'oil et le théâtre. La question du regard au tournant des XIX et XXe siècles sur les scènes européennes. Études théâtrales et études visuelles - Approches croisées. Sous la direction de Florence Baillet, Mireille Losco-Lena et Arnaud Rykner, Études théâtrales, n 65 , septembre 2017, $256 \mathrm{p}$.

Ce volume, qui réunit les actes d'un colloque international organisé à Paris en 2015, se distingue par l'originalité de son approche. Si le théâtre européen au tournant des $\mathrm{XIX}^{\mathrm{e}}$ et $\mathrm{XX}^{\mathrm{e}}$ siècles se caractérise par une «poussée du regard »- précisent Florence Baillet, Mireille Losco-Lena et Arnaud Rykner dans leur introduction -, qui se manifeste par un goût prononcé pour les effets spectaculaires et la profusion des images, il peut sembler pertinent d'interroger, non pas les manifestations du grand spectacle en elles-mêmes (qui ont déjà fait l'objet d'un bon nombre d'études sur la période), mais la manière dont elles ont engendré de nouvelles façons de regarder. Situant la réflexion au croisement des études théâtrales et des études visuelles (dont les auteurs montrent comment il a pu nourrir des réflexions fécondes dans le champ scientifique allemand et anglo-saxon), les directeurs scientifiques de cet ouvrage font ainsi œuvre innovante. En déplaçant le centre de gravité de l'analyse de l'image vers le regard, ils invitent à considérer le théâtre non plus seulement dans sa capacité à produire des images, mais comme un lieu où s'expriment un questionnement et un renouvellement des pratiques et des expériences du «voir ». Ils engagent la réflexion dans une perspective phénoménologique qui tente d'identifier la part du corps et du tactile dans le regard tout en cherchant à définir le tournant du $\mathrm{XIX}^{\mathrm{e}}-\mathrm{XX}^{\mathrm{e}}$ siècle comme une période cruciale où la vision connaît un changement de paradigme.

Divisé en quatre parties, l'ouvrage présente un ensemble de 14 études assez différentes de facture mais qui offre un ensemble cohérent. La première partie interroge les dispositifs et les nouvelles modalités du regard au XIX ${ }^{e}$ siècle. Ulrike Haß montre comment l'effondrement de l'architecture panoptique (type d'architecture carcérale imaginée par les frères Bentham à la fin du XVIII siècle afin de permettre à un gardien, logé dans une tour centrale, d'observer tous les prisonniers sans qu'ils se sachent observés) ouvre sur une crise de la visibilité, où « observation, représentation et réalité ne se rejoignent plus » (p. 26). Le panoptique, 
analysé par Michel Foucault comme "la métaphore de l'exercice du pouvoir dans les sociétés modernes » (p. 17), favorise un régime de visibilité où l'acte d'observer et celui d'être observé sont liés par un principe de confrontation. Sa disparition au cours du XIXe siècle cède le pas à de neuves pratiques d'observation que l'auteur identifie dans l'apparition de nouvelles scènes, tels le panorama et le "daguerréotype mobile », ou dans les travaux de Brecht et d'Appia. L'espace architectural du musichall, analysé par Stéphane Tralongo, permet d'identifier d'autres modalités du regard. Cet espace, élaboré pour favoriser la mobilité du spectateur, initie de nouvelles attitudes spectatorielles auxquelles répondent les « architectures de la fluidité » que l'auteur repère, entre autres, dans les romans de Zola. L'étude de Nic Leonhardt, qui sonde les modalités particulières du regard que supposent les images 3D et leur ancêtre le stéréoscope, clôt la première partie du volume.

Les deux parties centrales s'attachent à décrire deux régimes de visibilité décrits dans l'introduction comme « les plus symptomatiques du tournant 1900 au sein du théâtre » (p. 11) : le regard « intériorisé » et le regard " corporalisé ». Le premier ensemble s'intéresse au statut des images intérieures et à la nature d'un regard façonné par la vision onirique. On y trouve d'intéressantes études sur le « regard aveugle » dans le théâtre de W. B. Yeats (Pierre Longuenesse), sur l'usage de la gaze ou de la toile métallique sur les scènes de la fin du XIX ${ }^{\mathrm{e}}$ siècle, qui « floute pour mieux faire voir par projection intime » (p. 67) et change la nature même du regard (Arnaud Rykner), sur les leurres du grand spectacle dans les drames historiques mettant en scène la Révolution française à la Belle Époque (Sophie Lucet). Le deuxième ensemble questionne l'intégration du regard dans le domaine du sensible et de l'affect. La dramaturgie des images mise en place par le mélodrame à la fin du XVIIIe siècle (Kati Röttger), les fonctions de l'œil dans la pratique du jeu de l'acteur (Anne Pellois), l'élaboration d'un régime clinique du regard comme support d'une (re)définition de la mise en scène chez Firmin Gémier (Mireille Losco-Lena) sont autant d'études qui donnent à percevoir les différentes formes du regard «corporalisé ». Celui-ci est d'autant mieux circonscrit par l'étude de Florence Baillet qui, s'emparant du concept de « regard haptique » théorisé par Aloïs Riegl, montre de façon magistrale combien la dimension tactile est venue travailler la vision au XIX ${ }^{\mathrm{e}}$ siècle.

Pour clore cette riche analyse, il fallait encore explorer les zones limites où le regard s'engouffre dans le jeu de l'interdit. C'est l'objet de la dernière partie de l'ouvrage qui analyse le dispositif voyeuriste mis en place dans le genre de la revue (Romain Piana), l'engouement pour le spectacle du nu, de la mort, de l'exécution et du danger (Patrick Désile), les pantomimes frénétiques des frères Hanlon-Lees dans leur manière d'avoir transformé les perceptions (Ariane Martinez). Une ultime contri- 
bution fait œuvre de conclusion en analysant L'Atlas Mnémosyne d'Aby Warburg (1924-1929) et la mise en scène du prologue de Didon et Enée par Sasha Waltz (2005). Le regard « moderne », dont on a compris qu'il se caractérisait par une grande variété des pratiques et des expériences visuelles, trouve son ancrage théorique dans l'étude d'Alexander Jackob, qui en dévoile l'héritage à la fois sur la pratique du regard du spectateur et sur la construction des œuvres artistiques en elles-mêmes.

Cet ouvrage, au sujet audacieux, mérite ainsi l'attention. On pourra certes lui reprocher, à certains endroits, quelques approximations d'ordre historique ou méthodologique : l'iconographie rattachée au Chien de Montargis par exemple (p. 122 sq., ill. 5) n'illustre pas, contrairement à ce qui est écrit, le mélodrame de Pixerécourt en 1814 mais provient d'une étude sur la légende du chien de Montargis publiée dans la revue Lectures pour tous en 1902. Elle n'a donc pas de lien dans le mélodrame joué quelques décennies plus tôt. Cette erreur peut paraître problématique dans la mesure où l'article s'appuie sur l'iconographie du mélodrame pour montrer comment le genre est parvenu à initier un renouvellement du regard par sa mise en scène du corps en mouvement. On remarquera aussi quelques citations fautives (comme la célèbre formule de Charles Nodier présentant le mélodrame comme « la seule tragédie populaire qui convienne à notre époque » devenue, sans doute par le jeu des traductions successives, « le seul genre que la Révolution française ait vu naître », p. 117). Mais ces détails - que l'auteur des présentes lignes, en tant que spécialiste des formes théâtrales du premier XIX ${ }^{\mathrm{e}}$ siècle, peut repérer facilement - ne nuisent en rien à la qualité de cet ouvrage, qui montre de façon manifeste tout le bénéfice que l'on peut tirer des études visuelles pour l'analyse des œuvres théâtrales. Il est l'un des premiers à le faire dans le champ scientifique français, et réclame en cela tout l'intérêt des chercheurs.

Roxane Martin

Hélène Leclerc (dir.), Le Sud-Ouest de la France et les Pyrénées dans la mémoire des pays de langue allemande au XXe siècle, Le Pérégrinateur éditeur, Toulouse, 2018, 232 p.

L'idée de ce très bel ouvrage, à la présentation soignée, est née au cours d'un projet de recherche développé par Hilda Inderwildi et Hélène Leclerc au sein du Centre de Recherches et d'Études Germaniques (CREG) de l'université Toulouse-Jean-Jaurès. Sous forme de dictionnaire, il présente les portraits d'intellectuels, d'écrivains et d'artistes germanophones, originaires d'Allemagne, d'Autriche, de Suisse ou encore de régions autrefois sous la tutelle de l'Autriche-Hongrie, exilés en France et dont la plupart connurent les camps d'internement créés par la Troisième République au moment du déclenchement de la guerre et/ 
ou les camps de la période vichyssoise. Si le passage des Pyrénées par Walter Benjamin, guidé par Lisa Fittko, est un épisode connu, la naissance d'une « communauté d'antifascistes » à l'initiative du pédagogue allemand Pitt Krüger et de son épouse au pied des Pyrénées, au mas de Coûme en 1934 l'est peu : c'est l'intérêt de cet ouvrage qui lie moments déjà bien travaillés et portraits de personnes moins célèbres. Alors qu'on estime à quelque 55000 réfugiés du Troisième Reich en France pour la période 1939-1945, ce dictionnaire ne pouvait viser l'exhaustivité, les 79 portraits, tous accompagnés de textes d'auteur-e, tentent ainsi de trouver un équilibre dans la diversité des parcours biographiques, des statuts professionnels, des origines nationales, du degré de notoriété et aussi de la représentativité des femmes. Les portraits sont le fruit du travail de 28 contributeur-e-s, dont un nombre important est membre du CREG. On lira ou relira avec attention le texte de Jean Améry sur Gurs, un très beau passage du Diable en France de Feuchtwanger mais aussi la traduction, inédite, d'un extrait de l'autobiographie de Marta Feuchtwanger, Nur eine Frau. Jahre Tage Stunden, l'extrait de Voyage et destin où Alfred Döblin évoque la recherche qu'il entreprend pour retrouver sa femme et son fils à Toulouse, la lettre de Hannah Arendt à Günther Anders où elle évoque Gurs également, un extrait du Livre des Pyrénées de Kurt Tucholsky, du Roman d'Henri IV. La jeunesse du roi, d'Heinrich Mann ou Des morts restent jeunes d'Anna Seghers. On ne peut que saluer la parution de cet ouvrage et remercier son éditrice qui l'a dédié à la mémoire d'Annick Carlier, secrétaire scientifique de Germanica, disparue soudainement cette année. 
